'Unidad de Endoscopia, Hospital de Villarrica. Villarrica, Chile. ${ }^{2}$ Departamento de Gastroenterología, Pontificia Universidad Católica de Chile. Santiago, Chile.

${ }^{3}$ Escuela de Medicina, Pontificia Universidad Católica de Chile. Santiago, Chile.

${ }^{4}$ Unidad de Gastroenterología, Clínica Alemana de Santiago. Santiago, Chile.

${ }^{5}$ Departamento de Anatomía Patológica, Universidad de la Frontera. Temuco, Chile.

${ }^{6}$ Unidad de Gestión, Hospital de Villarrica. Villarrica, Chile. ${ }^{7}$ Southern Oxford School Villarrica. Villarrica, Chile.

Trabajo no recibió financiamiento. Los autores declaran no tener conflictos de interés.

Recibido el 24 de marzo de 2019, aceptado el 18 de noviembre de 2019.

Correspondencia a Dr. Luis Coppelli Villarroel Unidad de Endoscopía, Hospital de Villarrica. Villarrica, Chile. rcoppelli@hotmail.com

\section{La derivación protocolizada a endoscopía asociada a la detección de Helicobacter pylori mediante antígeno en deposiciones disminuye lista de espera para endoscopía y optimiza la detección de lesiones pre-malignas y cáncer gástrico incipiente}

\author{
LUIS COPPELLI ${ }^{1}$, LUIS ANTONIO DÍAZ ${ }^{2}$, ARNOLDO RIQUELME ${ }^{2}$, \\ CRISTIAN WAEGER ${ }^{3}$, ANTONIO ROLLÁN ${ }^{4}$, ENRIQUE BELLOLIO ${ }^{5}$, \\ JUAN CARLOS ARAYA ${ }^{5}$, MIGUEL ANGEL VILLASECA 5 , \\ MIGUEL VILLASMIL ${ }^{1}$, GONZALO PÉREZ ${ }^{6}$, CATALINA COPPELLI ${ }^{7}$
}

\section{Protocolized referral to endoscopy and Helicobacter pylori detected in stools aimed to decrease endoscopy waiting lists}

Background: Chile has one of the highest mortality rates by gastric cancer (GC) worldwide. Primary prevention of GC and detection of pre-neoplastic and early neoplastic lesions should be a national priority. Aim: To assess the impact of the protocolization of endoscopy referral and the use of $H$. pylori stool antigen test (HPSA) in the management of dyspepsia to decrease the waiting list for endoscopy and increase the detection of gastric pre-neoplastic and early neoplastic lesions. Material and Methods: We included all patients referred to the Endoscopy Unit of a regional hospital, from January 2015 to December 2017. We also included patients with known pre-neoplastic lesions and all those with first degree relatives with GC. We implemented protocols for referral of patients with dyspepsia considering the use of HPSA test, prioritizing to endoscopy those with a higher risk of GC. Results: A total of 4,641 endoscopies and 2,631 HPSA tests were carried out. After the adoption of these protocols, we observed a $52 \%$ decrease in the waiting time for endoscopy. The GC detection rate in this period was 1.8 to 3.1 cases per 100 endoscopies. After the adoption of the protocols, we observed a significant increase in early GC detection rate (from none in 2015 to 13\% in 2017, p = 0.03). Conclusions: The protocolization of the referral for endoscopy associated with widespread use of HPSA test in the management of patients with dyspepsia, are successful strategies to decrease waiting lists for endoscopy and optimize the detection rate of pre-neoplastic lesions and early GC.

(Rev Med Chile 2019; 147: 1382-1389)

Key words: Early Detection of Cancer; Endoscopy, Gastrointestinal; Gastritis, Atrophic; Helicobacter pylory; Stomach Neoplasms. 
El cáncer gástrico es la quinta causa más frecuente de cáncer y la tercera causa de muerte en este grupo de patologías, constituyendo un importante problema de salud en el mundo ${ }^{1}$. En particular, Chile cuenta con una de las tasas de mortalidad más altas a nivel mundial, constituyendo además la primera causa de muerte por cáncer en hombres ${ }^{2}$. La región de la Araucanía (IX) es una de las que cuenta con mayor mortalidad a nivel nacional por esta causa, con una tasa de 27,3 muertes por 100.000 habitantes.

Existen múltiples factores de riesgo asociados a cáncer gástrico, entre los cuales destaca la infección crónica por $H$. pylori (HP). De hecho, la Organización Mundial de la Salud categorizó al HP como un carcinógeno del grupo I en el año $1994^{3}$. El $73 \%$ de la población chilena cuenta con una infección crónica por esta bacteria, cifra bastante superior a la prevalencia estimada a nivel mundial ${ }^{4,5}$. La erradicación de HP ha demostrado resultados beneficiosos en dispepsia, linfoma MALT y adenocarcinoma gástrico ${ }^{6,7}$. De hecho, la erradicación en individuos con cáncer gástrico incipiente disminuye la incidencia de cáncer gástrico metacrónico en $50 \%{ }^{8}$. Asimismo, la erradicación en personas asintomáticas a demostrado disminuir la incidencia de cáncer gástrico en población asiática ${ }^{9}$, especialmente en sujetos sin atrofia gástrica, en los que la protección es casi absoluta ${ }^{10}$.

Aunque la detección precoz y prevención del cáncer gástrico ha sido reconocida como una prioridad de salud pública de acuerdo a la guía clínica MINSAL, existen serias limitaciones y desafíos en la prevención y manejo de esta patología. En Chile no existe un programa racional basado en la evidencia que permita enfrentar efectivamente el problema del cáncer gástrico.

En el Nodo Villarrica, el diagnóstico y tratamiento de la patología por HP y sus complicaciones asociadas se encontraba determinada por las guías MINSAL, las cuales sólo explicitan la erradicación en ciertas condiciones ${ }^{11,12}$ y proporcionan recomendaciones generales y poco específicas con respecto a test de diagnóstico no invasivos como el de aire espirado $\left(\mathrm{C}^{13} \mathrm{y} \mathrm{C}^{14}\right)$ o el antígeno de $H$. pylori en deposiciones (AHPD).

Hasta fines del año 2015, el diagnóstico de la infección por HP era realizado exclusivamente mediante test de ureasa y/o biopsias gástricas, ambos dependientes de la realización de una endoscopía digestiva alta (EDA), un procedimento costoso y escaso y no exento de riesgos. Los tests diagnosticos no invasivos de diagnóstico no se encontraban disponibles en la región. Tampoco había priorización de pacientes para endoscopia de acuerdo al riesgo estimado de cáncer gástrico, ni se hacía seguimiento endoscópico. La deficiencia de programas definidos y la falta crónica de recursos, favoreció el aumento de las listas de espera para EDA en nuestra región (2.000 pacientes aproximadamente con tiempos de espera de hasta 4 años). Además, esto desfavorecía a pacientes derivados por sospecha de patologías graves, quienes recibían, indudablemente, un diagnóstico tardío de su condición.

Basados en la realidad previamente descrita, nació la necesidad de crear un sistema protocolizado del manejo de la dispepsia a nivel de Atención Primaria de Salud (APS) usando el AHPD. Asimismo, se protocolizó la indicación de EDA y su realización de acuerdo a las guías actuales. El objetivo del presente trabajo fue evaluar el impacto de estas medidas en disminuir la lista de espera para endoscopía y como resultado de esto favorecer la detección de lesiones pre-malignas y cáncer gástrico precoz.

\section{Materiales y Métodos}

\section{Pacientes e intervenciones}

Se incluyeron a todos los pacientes derivados a la Unidad de Endoscopía del Hospital de Villarrica por dispepsia, desde el enero de 2016 hasta diciembre de $2017^{13}$. También se incluyeron pacientes con lesiones pre-malignas conocidas y aquellos con antecedentes de cáncer gástrico en familiar de primer grado.

A partir de enero del año 2016, se implementó un protocolo de manejo de pacientes dispépticos a nivel de todos los centros de atención primaria del nodo, que consideró el uso de test AHPD para el diagnóstico de infección por HP (NADAL ${ }^{\circledR} H$. pylori Antigen Test), como herramienta primaria de manejo en todos los pacientes con bajo riesgo de cáncer gástrico. La indicación de EDA se categorizó en precoz (urgente $\leq 7$ días) o en diferido (electiva $>7$ días) de acuerdo al riesgo basal del paciente. Las endoscopías se realizaron de acuerdo a las recomendaciones de la Asociación Chilena de Endoscopia Digestiva ${ }^{14}$. Se utilizaron equipos básicos de endoscopia diagnóstica (Fuji 530-590 y Pentax HD+| EG29-10). 


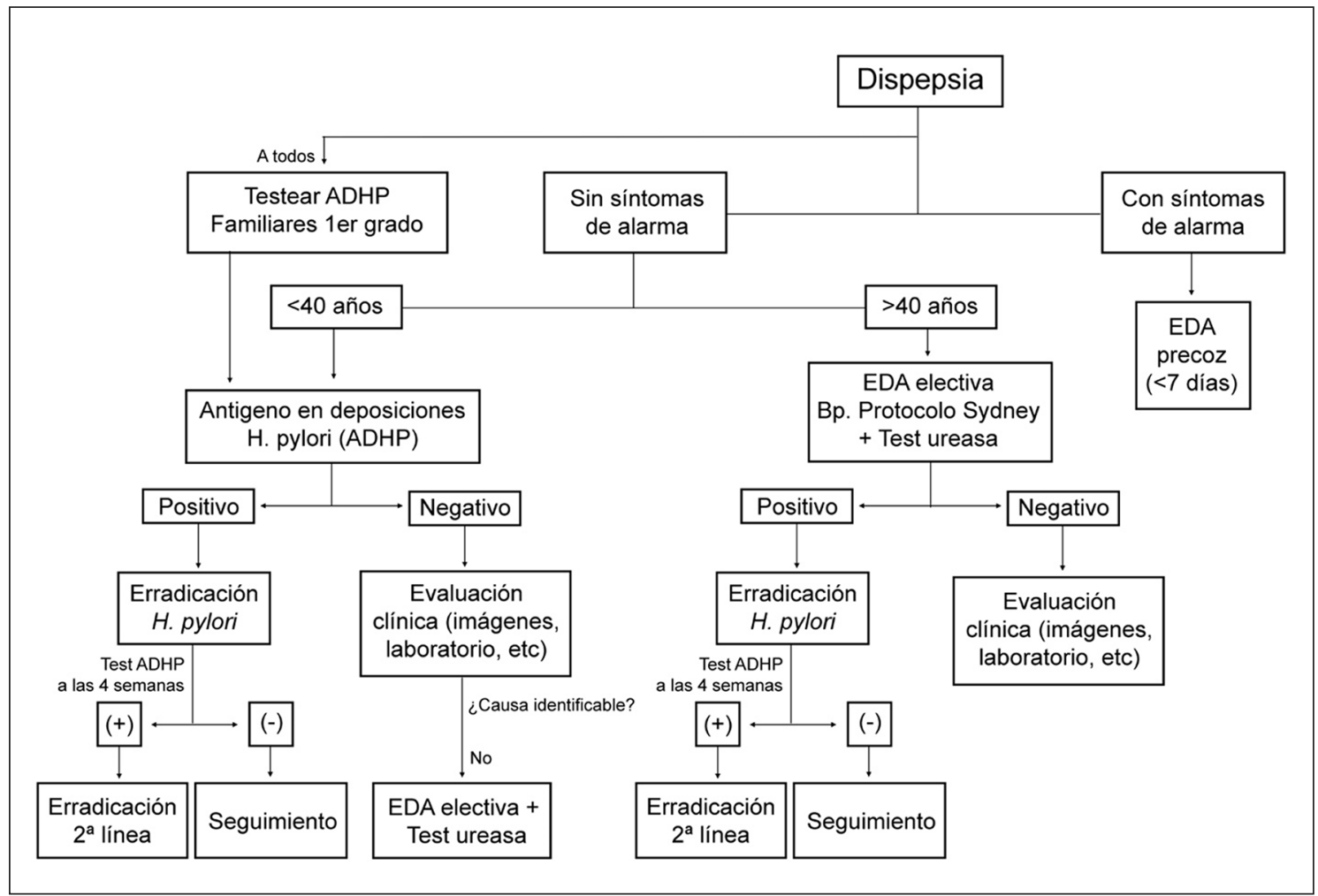

Figura 1. Algoritmo diagnóstico en pacientes con dispepsia de acuerdo a edad y presencia de síntomas de alarma.

Se establecieron programas educativos para médicos de APS para el uso de estos protocolos y de la correcta indicación de endoscopia. También se diseñaron panfletos educativos para pacientes y se elaboró un programa de educación dirigida a las autoridades de salud y la población en general con respecto a la infección por HP y sus consecuencias.

\section{Protocolos de manejo}

Los pacientes se estratificaron en 6 grupos de manejo (Figuras 1 y 2 ):

\section{Pacientes $<40$ años con dispepsia, sin sintomas de alarma}

Evaluación inicial con (ADHP: si es positivo se indica esquema de erradicación de primera línea, (de acuerdo a la guía MINSAL 2013), con control AHPD tras 4 semanas de finalizar erradicación. En aquellos persistentemente positivos se les erradica con esquema de segunda línea (omeprazol, amoxicilina, tetraciclina y bismuto). Todos quienes persisten sintomáticos se derivan a EDA con test

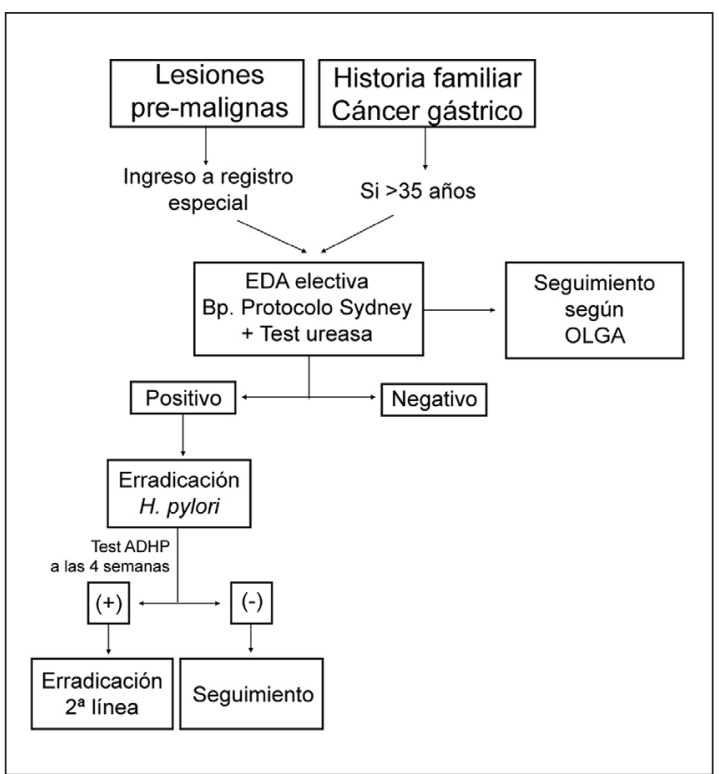

Figura 2. Algoritmo diagnóstico en pacientes con antecedentes de lesiones pre-malignas o antecedente familiar de primer grado con cáncer gástrico. 
de ureasa. Aquellos con test ADHP inicialmente negativo, se evalúan clínicamente con exámenes de laboratorio e imágenes ${ }^{15}$. Todos quienes persisten sin causa identificada se someten a EDA con test de ureasa.

\section{Pacientes $\geq 40$ años con dispepsia, sin sintomas de alarma}

EDA electiva, test de ureasa y biopsias según sistema Sydney. Biopsias analizadas de acuerdo a protocolo OLGA (Operative Link for Gastritis Assessment), de etapificación de riesgo de cáncer gástrico ${ }^{16,17}$. Se indica tratamiento de erradicación a todos los casos positivos. Control de erradicación con ADHP a las 4 semanas del seguimiento.

\section{Pacientes con dispepsia asociada a sintomas de alarma}

Pacientes que presentan:

a) Dolor abdominal epigástrico de origen no explicado que dura más de 2 semanas.

b) Más cualquiera de los síntomas listados a continuación: anemia ferropriva, disfagia, pérdida de peso no explicada, náusea persistente, saciedad precoz, hemorragia digestiva evidente u oculta, debilidad no explicada, masa abdominal o nódulo supraclavicular.

Todos estos pacientes se derivan para EDA urgente ( $<7$ días), y reciben tratamiento de acuerdo a los hallazgos endoscópicos y test de ureasa.

\section{Pacientes con lesiones pre-malignas}

Todos los pacientes en que se les identifican lesiones pre-malignas (gastritis atrófica, metaplasia intestinal y displasia), se incorporan a un registro especial y se realiza seguimiento endoscópico según la estratificación de riesgo ${ }^{14}$. Las endoscopías de control se realizan con toma de biopsias por sistema Sydney y análisis histológico según protocolo OLGA.

\section{Pacientes con cáncer gástrico en familiares de primer grado}

En los pacientes $\geq$ de 35 años se realiza EDA, test de ureasa, biopsias, etapificación según estadíos de OLGA, y seguimiento endoscópico de acuerdo a las recomendaciones del protocolo $\mathrm{ACHED}^{14}$. Se erradica a aquellos HP (+) y se controlan con ADHP. Adicionalmente, se controla con ADHP a los familiares de pacientes con test (+) (en aquellos $<35$ años erradicación del HP y los $>35$ años EDA, test de ureasa y biopsia).

6. En el caso de la población pediátrica, no se erradican pacientes con HP $(+)$ menores de 18 años asintomáticos. Aquellos sintomáticos, se derivan a pediatra para tratamiento de erradicación.

\section{Análisis de la información}

Los resultados fueron obtenidos a partir de la base de datos del Departamento de Gestión y de la Unidad de Endoscopía del Hospital Villarrica y fueron analizados de manera anónima. No se registró el uso previo o concomitante de inhibidores de la bomba de protones o antibioticos. Los resultados fueron expresados en promedio, desviación estándar o porcentajes. Se empleó $t$ de student o $\chi^{2}$ según corresponda. Se consideró significativo un valor $\mathrm{p}<0,05$. Se utilizó el software IBM SPSS v22.0 (Armonk, NY: IBM Corp.). Éste estudio fue aprobado por el Comité de Ética de la Pontificia Universidad Católica de Chile (ID: 180928003).

\section{Resultados}

Se incluyeron 4.641 endoscopías realizadas entre enero de 2015 y diciembre de 2017. En 83\% de las EDA se realizó test de ureasa (3.852 exámenes), $54 \%$ fueron positivos y $46 \%$ negativos ( 2.080 y 1.772 , respectivamente). A partir de enero del año 2016 se incorporó el ADHP al manejo de pacientes dispépticos. Durante los años 2016 y 2017 se realizaron 2.631 ADHP en el sistema público y privado del Nodo de Villarrica (Tabla 1).

A fines del año 2015 la lista de espera para EDA era de 1.964 pacientes. Tras la incorporación del protocolo, se observó una disminución progresiva de la lista de espera, con 1.420 pacientes el 2016 y 950 paciente el 2017 (disminución 51,6\% en 2 años). En ésta lista se incluyen aproximadamente 450 pacientes en seguimiento endoscópico de lesiones pre-malignas gástricas y otras patologías (esófago de Barrett, esofagitis intensa definida clasificación de Los Angeles, entre otras) ${ }^{18}$. Asimismo, tras la incorporación del protocolo el tiempo de espera disminuyó de 384 a 183 entre 2015 y 2017, respectivamente (disminución de 51,9\%) (Figura 3). Cabe destacar que al año 2017 el tiempo de espera de pacientes derivados con 
Tabla 1. Resumen de endoscopías y test de antígeno en deposiciones de $\boldsymbol{H}$. pylori realizados entre los años 2015 y 2017

\begin{tabular}{|cccc|}
\hline & $\begin{array}{c}\text { Endoscopías digestivas } \\
\text { altas }\end{array}$ & \multicolumn{2}{c|}{$\begin{array}{c}\text { Antígeno en deposiciones H. pylori } \\
\text { Laboratorios privados }\end{array}$} \\
\hline 2015 & 869 & 0 & 0 \\
2016 & 1.559 & 478 & 230 \\
\hline 2017 & 2.213 & 1.581 & 342 \\
\hline Total & 4.641 & & 2.631 \\
\hline
\end{tabular}

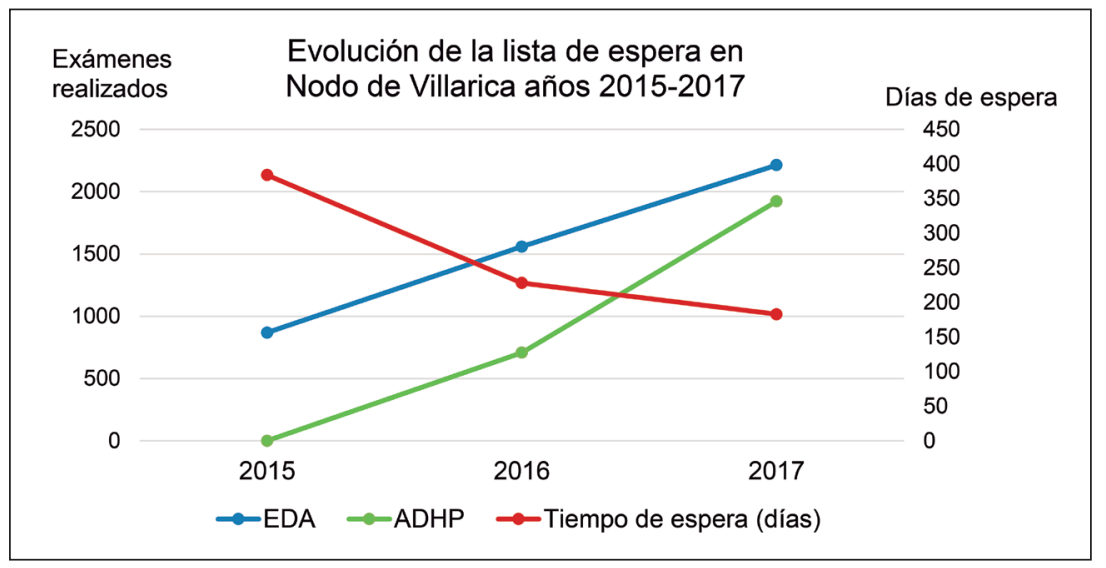

Figura 3. Evolución de la lista de espera en el Nodo de Villarrica comparado con el número de endoscopías digestivas altas y antígeno en deposiciones H. pylori para los años 2015 a 2017.

Tabla 2. Resumen de endoscopías y test de antígeno en deposiciones de $H$. pylori realizados entre los años 2015 y junio de 2017

\begin{tabular}{|llccc|}
\hline Endoscopias & & $\begin{array}{c}\text { Cáncer gástrico } \\
\text { avanzado }\end{array}$ & $\begin{array}{c}\text { Cáncer gástrico } \\
\text { incipiente }\end{array}$ & $\begin{array}{c}\text { Lesiones } \\
\text { pre-malignas }\end{array}$ \\
\hline 2015 enero-diciembre & $(n=869)$ & 27 & 0 & 0 \\
2016 enero-diciembre & $(n=1.559)$ & 30 & 2 & $536(34,38 \%)$ \\
2017 enero-junio & $(n=987)$ & 17 & 4 & $296(29,99 \%)$ \\
\hline
\end{tabular}

* Por limitaciones en el manejo información en el departamento de Gestión del Hospital la lista de espera para EDA para procedimientos electivos y de seguimiento es la misma; pacientes agendados electivamente para seguimiento no se pueden separar de aquellos casos nuevos. *Se han usados kits de diferentes laboratorios de acuerdo a licitaciones realizadas por el departamento de adquisiciones del Hospital de acuerdo a instrucciones del laboratorio.

síntomas de alarmas fue menor a 30 días. Por lo tanto, actualmente no hay pacientes con síntomas de alarma en lista de espera, ya que se derivan por un canal expedito a endoscopia.

Se evaluó la detección de cáncer gástrico incipiente y lesiones pre-maligmas mediante EDA entre enero de 2015 y junio de 2017 (Tabla 2). La tasa de cancer gástrico durante el período previa- mente descrito fluctuó entre 1,8 y 3,1 por cada 100 endoscopías. Tras instaurar estos protocolos, se observó un aumento significativo en la detección de cancer gástrico incipiente ( $0 \%$ el 2015 a 12,8\% de los casos el 2017, valor $\mathrm{p}=0,027)$. Asimismo, se detectaron 32,7 casos de lesiones premalignas por cada 100 endoscopías (no eran evaluadas dirigidamente el año 2015; valor $\mathrm{p}<0,0001$ ). 


\section{Discusión}

El consenso Maastricht V/Florence 2015 establece que la colonización del estómago por HP es una enfermedad infecciosa, incluso en ausencia de síntomas (nivel de evidencia $1 b$ y grado de recomendación A2 $)^{19}$. También propone la estrategia "test and treat strategy" como una opción válida en el manejo de la dispepsia no investigada (no aplicable a pacientes con síntomas de alarma o de mayor edad), con un alto nivel de evidencia y recomendación. Asimismo, recomienda descartar la gastritis por HP antes de establecer el diagnóstico de dispepsia funcional.

Basado en estos principios y considerando que la patología claramente sobrepasaba la modalidad de trabajo que se empleaba hasta entonces, se estableció un esquema operativo en relación a la patología asociada al Helicobacter y cáncer gástrico que llamamos "Cáncer gástrico, el programa del Hospital de Villarrica". Este programa se enfocó en tres aspectos: 1. Prevención primaria del cáncer gástrico (Erradicación del H. pylori; y educación de la población, autoridades y estamento médico en relación a esta patología); 2 . Manejo de pacientes con historia familiar de cáncer gástrico; 3 . Detección de lesiones pre-malignas (y su seguimiento en el tiempo), e identificación de lesiones precoces.

Como resultado del programa, se observó una disminución de 51,9\% de tiempo en la lista de espera para endoscopia, cifra que incluye el considerable número de pacientes alistados para seguimiento endoscópico. Por ende, aquellos derivados por sintomatología y/o factores de riesgo, no deben esperar más de 30 días para realizarse endoscopía. Estos resultados se deben al conjunto de intervenciones que incluyó la derivación protocolizada de pacientes con mayor riesgo de cáncer y el uso del ADHP en el manejo de la dispepsia en pacientes de bajo riesgo de cáncer gástrico. En efecto, dado que el AHPD tiene una sensibilidad y especificidad mayor a $90 \%$, es de bajo costo y sin complicaciones, consideramos que su uso tendrá un alto impacto en el sistema público ${ }^{10}$. Como evidencia de esto, observamos que el uso del AHPD nos permitió ahorrar 2.631 endoscopías entre el 2016 y 2017 , lo que equivale a $69,8 \%$ de las endoscopías realizadas en ese período.

La región de la Araucanía Sur, donde se encuentra la ciudad de Villarrica tiene una mortalidad por cáncer gástrico de 27,3 por 100.000 habitantes, que es mayor a la tasa nacional. Observamos que la instauración del protocolo permitió detectar lesiones pre-malignas en 32,7\% de todas las endoscopías, lo cual es una cifra ampliamente mayor a la realidad previa (estas lesiones no eran buscadas dirigidamente el 2015). Como grupo, nos parece que estas cifras reflejan la gran importancia en la selección de pacientes derivados a endoscopía, puesto que los cupos para endoscopía utilizados previamente por pacientes jóvenes con bajo riesgo de cáncer gástrico, han sido reemplazados por pacientes con mayor riesgo. Asimismo, este protocolo ha permitido contar con espacio para seguimiento endoscópico de pacientes con lesiones pre-malignas, estrategia que ha sido recientemente validada prospectivamente en la detección del cáncer gástrico ${ }^{21}$.

Considerando la evidencia previamente expuesta y comparando los costos de cada examen (AHPD US\$ 6 versus Endoscopía US\$150), nos parece de vital importancia incorporar estrategias de selección de pacientes con test de HP no invasivos en las Guías Clínicas MINSAL, junto con financiar el uso AHPD en el sistema público. Respecto a nuestra realidad local, se hizo presión a las autoridades de salud regionales para la adquisición y distribución del test fecal no solamente al hospital base en Villarrica, sino también a los consultorios y laboratorios privados, cosa que ha sido difícil, ya que el test fecal no está incluido en la canasta básica de prestaciones de salud de las APS. Al montarse el AHPD en los consultorios permitirá realizar un diagnóstico de la presencia de $\mathrm{HP}$ a edades más tempranas, en forma no invasiva, de bajo costo y sin endoscopía. Su amplio uso podría beneficiar no sólo a pacientes con cáncer gástrico, sino también pacientes con dispepsia y úlceras duodenales.

$\mathrm{Al}$ momento de instaurar los protocolos en todos los consultorios del Nodo, se dictaron charlas y módulos educativos a todos los médicos de APS para facilitar y promover su uso. También se organizó un programa educativo para pacientes y la población general, el cual ha tenido una aceptación importante y percepción positiva por parte de nuestros usuarios.

En relación a las cepas de HP, cabe destacar que no se evaluó la producción de toxinas, ya que la estrategia test and treat considera la erradicación independiente del grado de virulencia. Esta conducta está basada en el consenso de Maastrich V/ 
Florence, considerando nuestra alta incidencia de cáncer gástrico ${ }^{19,22-25}$. Sin embargo, consideramos importante evaluar la prevalencia de cepas con mayor virulencia, por lo que, actualmente se encuentra en curso un estudio colaborativo con la Universidad de la Frontera para dar respuesta a esta interrogante. Finalmente, respecto a los desafíos futuros, esperamos evaluar el impacto de estos cambios a largo plazo en el Nodo de Villarrica como grupo de investigación, especialmente en relación a la mortalidad y la incidencia de cáncer gástrico incipiente y avanzado.

\section{Conclusión}

La instauración de protocolos de derivación junto al amplio uso del antígeno de $H$. pylori en deposiciones, han resultado ser estrategias exitosas para disminuir la lista de espera en nuestra Unidad de Endoscopía y optimizar la detección y seguimiento de lesiones pre-malignas. Sin duda, esperamos que el impacto de esta estrategia se vea reflejado en la detección de mayor número de lesiones precoces y eventualmente lleguemos a reducir la mortalidad de cáncer gástrico en el Nodo de Villarrica a largo plazo.

\section{Referencias}

1. Ervik M, Lam F, Ferlay J, Mery L, Soerjomataram I, Bray F. Cancer Today. Lyon, France: International Agency for Research on Cancer. Cancer Today. 2016 [Available from: http://gco.iarc.fr/today.

2. Salud Md. Departamento de estadísticas e información de salud: Defunciones y Mortalidad por causas 2011 [Available from: http://www.deis.cl/defunciones-y-mortalidad-por-causas/.

3. Schistosomes, liver flukes and Helicobacter pylori. IARC Working Group on the Evaluation of Carcinogenic Risks to Humans. Lyon, 7-14 June 1994. IARC monographs on the evaluation of carcinogenic risks to humans 1994; 61: 1-241.

4. Ferreccio C, Rollán A, Harris PR, Serrano C, Gederlini A, Margozzini P, et al. Gastric cancer is related to early Helicobacter pylori infection in a high-prevalence country. Cancer Epidemiol Biomarkers Prev 2007; 16 (4): 662-7.

5. Hu Y, Zhu Y, Lu NH. Novel and Effective Therapeutic Regimens for Helicobacter pylori in an Era of Increasing
Antibiotic Resistance. Front Cell Infect Microbiol 2017; 7: 168 .

6. Wotherspoon AC, Doglioni C, Diss TC, Pan L, Moschini A, de Boni M, et al. Regression of primary low-grade B-cell gastric lymphoma of mucosa-associated lymphoid tissue type after eradication of Helicobacter pylori. Lancet (London, England) 1993; 342 (8871): 575-7.

7. Chey WD, Leontiadis GI, Howden CW, Moss SF. ACG Clinical Guideline: Treatment of Helicobacter pylori Infection. Am J Gastroenterol 2017; 112 (2): 212-39.

8. Choi IJ, Kook MC, Kim YI, Cho SJ, Lee JY, Kim CG, et al. Helicobacter pylori Therapy for the Prevention of Metachronous Gastric Cancer. N Engl J Med 2018; 378 (12): 1085-95.

9. Ford AC, Forman D, Hunt R, Yuan Y, Moayyedi P. Helicobacter pylori eradication for the prevention of gastric neoplasia. Cochrane Database Syst Rev. 2015; (7): Cd005583.

10. Wong BC, Lam SK, Wong WM, Chen JS, Zheng TT, Feng RE, et al. Helicobacter pylori eradication to prevent gastric cancer in a high-risk region of China: a randomized controlled trial. JAMA 2004; 291 (2): 187-94.

11. Salud Md. Guía Clínica Tratamiento de erradiación de Helicobacter pylori en el paciente con úlcera péptica. Santiago, Chile. 2013.

12. Salud Md. Guía Clínica AUGE Cáncer Gástrico. Santiago, Chile. 2014.

13. Drossman DA. Functional Gastrointestinal Disorders: History, Pathophysiology, Clinical Features and Rome IV. Gastroenterology. 2016.

14. Rollán A, Cortés P, Calvo A, Araya R, Bufadel ME, González R, et al. [Recommendations of the Chilean Association for Digestive Endoscopy for the management of gastric pre-malignant lesions]. Rev Med Chile 2014; 142 (9): 1181-92.

15. Moayyedi PM, Lacy BE, Andrews CN, Enns RA, Howden CW, Vakil N. ACG and CAG Clinical Guideline: Management of Dyspepsia. Am J Gastroenterol 2017; 112 (7): 988-1013.

16. Rugge $M$, Genta RM. Staging and grading of chronic gastritis. Hum Pathol 2005; 36 (3): 228-33.

17. Rugge M, Correa P, Di Mario F, El-Omar E, Fiocca R, Geboes K, et al. OLGA staging for gastritis: a tutorial. Dig Liver Dis 2008; 40 (8): 650-8.

18. Lundell LR, Dent J, Bennett JR, Blum AL, Armstrong D, Galmiche JP, et al. Endoscopic assessment of oesophagitis: clinical and functional correlates and further validation of the Los Angeles classification. Gut 1999; 45 (2): 172-80.

19. Malfertheiner P, Megraud F, O’Morain CA, Gisbert JP, Kuipers EJ, Axon AT, et al. Management of Helicobacter 
pylori infection-the Maastricht V/Florence Consensus Report. Gut 2017; 66 (1): 6-30.

20. Scuderi G, Celi D, Romagnolo M, Lupi A, Alecci L, Dramissino I, et al. Helicobacter pylori: use of a stool antigen detection test and a six-day triple therapy. Dig Liver Dis 2000; 32 (1): 71-3.

21. Rugge M, Meggio A, Pravadelli C, Barbareschi M, Fassan $\mathrm{M}$, Gentilini M, et al. Gastritis staging in the endoscopic follow-up for the secondary prevention of gastric cancer: a 5-year prospective study of 1755 patients. Gut. 2018.

22. McColl KE, Murray LS, Gillen D, Walker A, Wirz A, Fletcher J, et al. Randomised trial of endoscopy with testing for Helicobacter pylori compared with non-invasive $\mathrm{H}$ pylori testing alone in the management of dyspepsia. BMJ (Clinical research ed). 2002; 324 (7344): 999-1002.
23. Arents NL, Thijs JC, van Zwet AA, Oudkerk Pool M, Gotz JM, van de Werf GT, et al. Approach to treatment of dyspepsia in primary care: a randomized trial comparing "test-and-treat" with prompt endoscopy. Arch Intern Med 2003; 163 (13): 1606-12.

24. Lassen AT, Hallas J, Schaffalitzky de Muckadell OB. Helicobacter pylori test and eradicate versus prompt endoscopy for management of dyspeptic patients: 6.7 year follow up of a randomised trial. Gut 2004; 53 (12): 1758-63.

25. Duggan AE, Elliott CA, Miller P, Hawkey CJ, Logan RF. Clinical trial: a randomized trial of early endoscopy, Helicobacter pylori testing and empirical therapy for the management of dyspepsia in primary care. Aliment Pharmacol Ther 2009; 29 (1): 55-68. 